\title{
A non-convex regularization approach for compressive sensing
}

\author{
Ya-Ru Fan · Alessandro Buccini · Marco \\ Donatelli · Ting-Zhu Huang
}

Received: date / Accepted: date

\begin{abstract}
Compressive sensing (CS) aims at reconstructing high dimensional data from a small number of samples or measurements. In this paper, we propose the minimization of a non-convex functional for the solution of the CS problem. The considered functional incorporates informations on the selfsimilarity of the image by measuring the rank of some appropriately constructed matrices of fairly small dimensions. However, since the rank minimization is a NP hard problem, we consider, as a surrogate function for the rank, a non-convex, but smooth function. We provide a theoretical analysis of the proposed functional and develop an iterative algorithm to compute one of its stationary points. We prove the convergence of such algorithm and show, with some selected numerical experiments, that the proposed approach achieves good performances, even when compared with the state of the art.
\end{abstract}

The work of the first and the fourth author is supported by 973 Program (2013CB329404), NSFC (61370147). The work of the second and the third author is supported in part by MIUR - PRIN 2012 N. 2012MTE38N and by a grant of the group GNCS of INdAM.

Y.-R. Fan

School of Mathematical Sciences,

University of Electronic Science and Technology of China,

Chengdu, China

E-mail: yarufanfan@163.com

A. Buccini

Kent State University,

Kent, Ohio, USA

E-mail: abuccini@kent.edu

M. Donatelli

University of Insubria,

Como, Italy

E-mail: marco.donatelli@uninsubria.it

T.-Z. Huang

University of Electronic Science and Technology of China,

Chengdu, China

E-mail: tingzhuhuang@126.com 
Keywords Compressive sensing · non-convex low-rank regularization · smoothed rank function

Mathematics Subject Classification (2010) 90C26 $65 \mathrm{~K} 10 \cdot 94 \mathrm{~A} 12$

\section{Introduction}

The theory of compressive sensing (CS) [11,17] asserts that sparse signals can be exactly reconstructed from a very small number of samples or measurements. A key condition behind the exact recovery for CS is the sparsity. In practice, many signals can be represented in some domain with most generating coefficients close to or equal to zero. In recent years, CS has received considerable attention, thanks to the rapid grow of new applications which required the development of several reliable and computationally efficient numerical methods. A few of the potential CS-based applications are medical image reconstruction [26, 27], image acquisition [20,31, and sensor networks 30].

The CS recovery problem seeks the perfect reconstruction of a signal $\mathbf{x} \in$ $\mathbb{R}^{N}$ from $M$ linear measurements

$$
\mathbf{y}=\Phi \mathbf{x}
$$

where $\Phi \in \mathbb{R}^{M \times N}$ is the measure matrix. We assume that $\mathbf{y}$ is attainable, i.e., that $\mathbf{y}$ lies in the range of $\Phi$, and that $M \ll N$. Under these assumptions, it holds that there exists more than one $\mathbf{x}$ that satisfies the linear equation $\mathbf{y}=\Phi \mathbf{x}$. The standard CS recovery technique aims at recovering the unknown signal by finding the sparsest $\mathbf{x}$ such that $\mathbf{y}=\Phi \mathbf{x}$, i.e., it looks for the solution of the minimization problem

$$
\arg \min _{\mathbf{x}}\|\mathbf{x}\|_{0} \quad \text { s.t. } \quad \mathbf{y}=\Phi \mathbf{x}
$$

where $\|\cdot\|_{0}$ denotes the so-called $\ell_{0}$-norm. We recall that the $\ell_{0}$-norm is not a norm and $\|\mathbf{x}\|_{0}$ is defined as the number of nonzero elements of $\mathbf{x}$. Since $\ell_{0}$-norm minimization is a NP-hard and non-convex problem, it is difficult to computationally solve (11). Conventional CS recovery replaces the $\ell_{0}$-norm by the $\ell_{1}$-norm (denoted by $\|\cdot\|_{1}$ ) in (1) leading to a convex optimization problem, namely,

$$
\arg \min _{\mathbf{x}}\|\mathbf{x}\|_{1} \quad \text { s.t. } \quad \mathbf{y}=\Phi \mathbf{x},
$$

which is the well-known basis pursuit problem [11.

In practical applications it is impossible to avoid the presence of the noise, thus only a noise contaminated version of $\mathbf{y}$, denoted by $\mathbf{y}^{\epsilon}$, such that

$$
\left\|\mathbf{y}-\mathbf{y}^{\epsilon}\right\|<\epsilon,
$$


is available. Here and throughout this paper, we will denote by $\|\cdot\|$ the Frobenius norm, which reduces to the $\ell_{2}$-norm when applied to vectors. In this setting, it is possible to rewrite problem (2) using an appropriate regularization parameter $\lambda>0$ as follows

$$
\arg \min _{\mathbf{x}}\left\|\mathbf{y}^{\epsilon}-\Phi \mathbf{x}\right\|^{2}+\lambda\|\mathbf{x}\|_{1}
$$

Since the minimization problem (44) is convex, there are many methods to efficiently solve it, including iterative shrinkage algorithm [3], alternating direction method of multipliers (ADMM) [5,23] and Bregman split algorithm [7, 8. 33.

Many practical problems of importance are non-convex and most nonconvex problems are difficult to solve in a reasonable time. In 13 it was proven that better performances for CS signal recovery can be achieved by replacing the $\ell_{1}$-norm with a non-convex $\ell_{p}$-norm $(0<p<1)$ though at the cost of higher computational complexity. Another possible approach for the solution of the $\ell_{p}$ problem has been proposed in [25], where a stationary point of the non-convex $\ell_{2}-\ell_{p}$ functional is computed by the computation of successive quadratic tangent majorants and projection in generalized Krylov subspaces.

Recent results suggest that better signal reconstruction can be obtained by means of the nonlocal sparsity with respect to the ones obtained using the global sparsity [28, 29]. In [19], a non-convex and low-rank regularization based model that exploited nonlocal sparsity is proposed for CS image (signal) recovery. The model stemmed from the observation that by partitioning an image in small patches, it is possible to find several patches with similar structure. We refer to this property as to self-similarity. It is possible to assume that, if those patches are column-stacked and collected in a matrix, that matrix will be of low-rank. To exploit this property, in [19] the image is subdivided in patches of fixed dimension and, for each exemplary patch, the $m$ most similar patches are collected into a matrix. Let us consider the $i$-th patch $\hat{\mathbf{x}}_{i}$, the collection of the $m$ most similar patches is denoted by

$$
P_{\mathbf{x}}^{(i)}=\left[P_{\mathbf{x}}^{\left(i_{0}\right)}, P_{\mathbf{x}}^{\left(i_{1}\right)}, \cdots, P_{\mathbf{x}}^{\left(i_{m-1}\right)}\right],
$$

where $P_{\mathbf{x}}^{\left(i_{j}\right)}$ is the column stacked $j$-th most similar patch to $\hat{\mathbf{x}}_{i}$ in the image $\mathbf{x}$. Under the assumption of self-similarity we obtain that $P_{\mathbf{x}}^{(i)}$ is a low-rank matrix.

To enforce the low-rank property, in [19], an accurate approximation for the rank of $A \in \mathbb{R}^{n \times m}$ is obtained by the smoothed rank function (SRF)

$$
G_{\delta}(A)=r-\sum_{j=1}^{r} \mathrm{e}^{-\sigma_{\mathrm{j}}^{2} / 2 \delta^{2}},
$$

where $\sigma_{j}$ is the $j$-th singular value of $A, r=\min \{n, m\}$, and $\delta$ is an adjustable parameter. Observe that the function $G_{\delta}(A)$ in (5) is positive and differentiable. 
In [19] the following minimization problem was proposed

$$
(\hat{\mathbf{x}}, \hat{\mathfrak{L}})=\arg \min _{\mathbf{x}, \mathfrak{L}}\left\|\mathbf{y}^{\epsilon}-\Phi \mathbf{x}\right\|^{2}+\eta \sum_{i}\left\{\left\|P_{\mathbf{x}}^{(i)}-L_{i}\right\|^{2}+\lambda G_{\delta}\left(L_{i}\right)\right\},
$$

where $\mathfrak{L}$ denotes the collection of the $L_{i}$ and $\eta, \lambda>0$ are regularization parameters. The first term in (6) is the fidelity term for the CS recovery problem and the remaining act as non-convex low-rank regularization terms. The latter was constructed to take into account the self-similarity of the image and enforce the low-rank property on the group patches.

In [19] the authors proposed an algorithm for tackling (6) which achieves very good numerical performances. Nevertheless, a theoretical analysis of the problem itself and of the resolving algorithm was missing.

In this paper, we propose a new CS image recovery method based on (6), which keeps its good properties, but with small changes such that a complete theoretical analysis can be provided. Namely, we slightly modify the functional in (6) for ensuring the existence of global minimizers, which we prove to be stable. Moreover, we show that the minimization of the modified functional induces a regularization method as defined in [18]. This last result ensures that, as the norm of the noise goes to 0 the global minimum of the functional converges to a solution of the problem. Thus, when the norm of the noise is not too large, the minimum of the functional is an accurate approximation of the solution of the original problem. The theoretical proof of the exact recovery property, i.e., the property that states that, with high probability, the considered approach reconstructs the exact solution of the problem, see, e.g., 1, 2, 10, 12, is still an open problem. Nevertheless, the computed numerical examples prove heuristically that the proposed approach is able to provide accurate reconstructions.

We then construct an algorithm based on the alternating descent strategy, for the computation of a stationary point and prove its convergence.

Several numerical results for uniform and pseudo-radial sampling of Fourier coefficients, prove that the modifications introduced in the model problem (6), which allow us to derive a complete theoretical framework, have not deteriorated the quality of the computed solutions which remain comparable with those obtained with the algorithm in [19]. Finally, even if the interest of this paper is on CS, the proposed method can be applied to other image recovery problems as well.

This paper is organized as follows: in Section 2 we present the proposed model for CS image recovery, Section 3 shows the theoretical results on the proposed model described above, in Section 4 we describe an algorithm for the computation of a stationary point of the proposed functional. Section 5 is devoted to numerical experiments and in Section 6 we draw some conclusions. 


\section{The proposed model}

First, we present the usage of nonlocal sparsity by patch grouping, which characterizes the self-similarity of an image. For the test image $\mathbf{x}$, we assume that it contains abundant self-similarity, which implies that a large number of similar patches can be found for any exemplary patch of size $\sqrt{n} \times \sqrt{n}$ at position $i$. We denote the $i$-th patch by $\hat{\mathbf{x}}_{i} \in \mathbb{R}^{n}$. As in [19], we use a variant of k-nearest-neighbor search in a local window for each exemplary patch $\hat{\mathbf{x}}_{i}$ to find its similar patches. Let $G_{i}$ be defined by

$$
G_{i}=\left\{i_{j} \mid\left\|\hat{\mathbf{x}}_{i}-\hat{\mathbf{x}}_{i_{j}}\right\| \leq c\right\},
$$

where $c>0$ is a pre-defined threshold, thus $G_{i}$ collects the positions of the corresponding similar patches. After this patches grouping, for the $i$-th exemplar patch $\hat{\mathbf{x}}_{i}$, we order its similar patches as column vectors lexicographically and form a matrix

$$
X_{i}=\left[\hat{\mathbf{x}}_{i}, \hat{\mathbf{x}}_{i_{1}}, \cdots, \hat{\mathbf{x}}_{i_{m-1}}\right] \in \mathbb{R}^{n \times m} .
$$

According to the above assumption, we know that each matrix $X_{i}$ is of lowrank. Thus, we can minimize the rank of the matrix $X_{i}$ to enhance the selfsimilarity, thus converting the nonlocal self-similarity to a rank minimization problem.

In fact, the resulting matrix $X_{i}$ inherits the noise from the corrupted image. To reconstruct a clean image, we let

$$
X_{i}=L_{i}+W_{i}
$$

where $L_{i}$ and $W_{i}$ denote the low-rank matrix and the Gaussian noise matrix, respectively. The low-rank matrix $L_{i}$ can be approximated by solving the following problem:

$$
\min _{L_{i}} \operatorname{rank}\left(L_{i}\right) \quad \text { s.t. } \quad\left\|X_{i}-L_{i}\right\|^{2} \leq \epsilon^{2}
$$

where $\epsilon$ is the residual error defined in (3). It is well known that problem (8) is NP-hard. To obtain an approximated solution, many works proposed the nuclear norm as a convex relaxation for the rank. The rank minimization problem using the nuclear norm can be solved efficiently by the singular value thresholding algorithm 6]. However, the nuclear norm minimization equally regularizes each singular value, which leads to a suboptimal performance since the singular values have different physical meanings and consequently should be treated differently [22]. Many CS studies have shown that non-convex lowrank approximation yields better results than convex ones. In [19, it was shown that the smoothed rank function, as non-convex surrogate for the rank, is able to obtain better performances than the nuclear norm.

Thus, a good approximation of the solution of (8) can be computed by

$$
\min _{L_{i}} G_{\delta}\left(L_{i}\right) \quad \text { s.t. } \quad\left\|X_{i}-L_{i}\right\|^{2} \leq \epsilon^{2},
$$


where $G_{\delta}\left(L_{i}\right)$ is the SRF defined in (5D), see 19] for more details. Since $G_{\delta}\left(L_{i}\right)$ is smooth, the above problem can be tackled by the gradient descent method.

In this paper, we focus on the CS image recovery, but the proposed method can be applied to other image recovery problems as well. Now we propose a CS based method, where the constrained problem (9) is treated as a low-rank unconstrained regularization, namely,

$$
\begin{gathered}
(\hat{\mathbf{x}}, \hat{\mathfrak{L}})=\arg \min _{\mathbf{x}, \mathfrak{L}}\|\mathbf{y}-\Phi \mathbf{x}\|^{2}+\eta \sum_{i}\left(\left\|P_{\mathbf{x}}^{(i)}-L_{i}\right\|^{2}+\lambda G_{\delta}\left(L_{i}\right)\right) \\
+\mu^{(1)}\|\mathbf{x}\|^{2}+\mu^{(2)} \sum_{i}\left\|L_{i}\right\|^{2},
\end{gathered}
$$

where $\eta, \lambda, \mu^{(1)}, \mu^{(2)}>0$ are regularization parameters. The model (10) employs the nonlocal sparsity via patch group and non-convexity of the SRF. The difference between (10) and (6) is the introduction of the last two terms, which are crucial for the following theoretical analysis.

Before moving to the theoretical analysis we would like to provide more details on the construction of $P_{\mathbf{x}}^{(i)}$. Let $m>1$ be a fixed number and let $\hat{\mathbf{x}}_{i}$ be a reference patch. Denote by $\tilde{P}_{\mathbf{x}}^{\left(i_{0}\right)}, \ldots, \tilde{P}_{\mathbf{x}}^{\left(i_{m-1}\right)}$ the column stacked $m$ most similar patches to $\hat{\mathbf{x}}_{i}$, i.e., $i_{0}, \ldots i_{m-1} \in G_{i}$, where $G_{i}$ is defined in (7). We define $m_{j}$ as the number of times that the patch $\hat{\mathbf{x}}_{j}$ is recognized as similar to another patch plus one. For example, if the patch $\hat{\mathbf{x}}_{1}$ is recognized as similar to patches $\hat{\mathbf{x}}_{3}, \hat{\mathbf{x}}_{5}, \hat{\mathbf{x}}_{42}$ then $m_{1}=4$. We can now construct $P_{\mathbf{x}}^{(i)}$ as

$$
P_{\mathbf{x}}^{(i)}=\left[\frac{1}{m_{i_{0}}} \tilde{P}_{\mathbf{x}}^{\left(i_{0}\right)}, \ldots, \frac{1}{m_{i_{m-1}}} \tilde{P}_{\mathbf{x}}^{\left(i_{m-1}\right)}\right]=\left[P_{\mathbf{x}}^{\left(i_{0}\right)}, \ldots, P_{\mathbf{x}}^{\left(i_{m-1}\right)}\right] .
$$

By doing so we avoid giving too much weight on patches that are be counted several times. Moreover, as we will see in the following, this helps us in the computations.

Note that the map which associate $\mathbf{x}$ with $P_{\mathbf{x}}^{(i)}$ is weakly lower semicontinuous since it can be written as a composition of indicator functions of closed sets.

\section{Theoretical analysis of the model}

We now provide a theoretical analysis of the properties of the proposed model (10). We denote the objective function by

$$
\begin{aligned}
J_{\eta, \lambda, \mu^{(1)}, \mu^{(2)}}^{\epsilon}(\mathbf{x}, \mathfrak{L})= & \left\|\mathbf{y}^{\epsilon}-\Phi \mathbf{x}\right\|^{2}+\eta \sum_{i}\left(\left\|P_{\mathbf{x}}^{(i)}-L_{i}\right\|^{2}+\lambda G_{\delta}\left(L_{i}\right)\right) \\
& +\mu^{(1)}\|\mathbf{x}\|^{2}+\mu^{(2)} \sum_{i}\left\|L_{i}\right\|^{2}
\end{aligned}
$$

and we prove a preliminary result on $J_{\eta, \lambda, \mu^{(1)}, \mu^{(2)}}^{\epsilon}$. 
Lemma 1 The functional $J_{\eta, \lambda, \mu^{(1)}, \mu^{(2)}}^{\epsilon}$ defined in (12) is positive, weakly lower semi-continuous, and coercive.

Proof By construction $J_{\eta, \lambda, \mu^{(1)}, \mu^{(2)}}^{\epsilon}$ is a sum of positive and weakly lower semicontinuous functions, thus the only thing that we need to prove is its coercivity.

We have to show that $J_{\eta, \lambda, \mu^{(1)}, \mu^{(2)}}^{\epsilon}(\mathbf{x}, \mathfrak{L}) \rightarrow \infty$ as $\|(\mathbf{x}, \mathfrak{L})\|^{2}=\|\mathbf{x}\|+$ $\sum_{i}\left\|L_{i}\right\|^{2} \rightarrow \infty$. This trivially follows from

$$
J_{\eta, \lambda, \mu^{(1)}, \mu^{(2)}}^{\epsilon}(\mathbf{x}, \mathfrak{L}) \geq \mu^{(1)}\|\mathbf{x}\|^{2}+\mu^{(2)} \sum_{i}\left\|L_{i}\right\|^{2} \rightarrow \infty \text { as }\|(\mathbf{x}, \mathfrak{L})\|^{2} \rightarrow \infty,
$$

since $\mu^{(1)}, \mu^{(2)}>0$.

We are now in the position to prove the existence of global minimizers for $J_{\eta, \lambda, \mu^{(1)}, \mu^{(2)}}^{\epsilon}$.

Theorem 1 (Existence) The functional $J_{\eta, \lambda, \mu^{(1)}, \mu^{(2)}}^{\epsilon}$ defined in (12) admits at least one global minimizer.

Proof Since $J_{\eta, \lambda, \mu^{(1)}, \mu^{(2)}}^{\epsilon}$ is positive, proper, and coercive, in force of Lemma 1 there exists $(\mathbf{x}, \mathfrak{L}) \in \mathcal{D}\left(J_{\eta, \lambda, \mu^{(1)}, \mu^{(2)}}^{\epsilon}\right)$ such that $J_{\eta, \lambda, \mu^{(1)}, \mu^{(2)}}^{\epsilon}(\mathbf{x}, \mathfrak{L})<\infty$. Let

$$
\nu=\inf \left\{J_{\eta, \lambda, \mu^{(1)}, \mu^{(2)}}^{\epsilon}(\mathbf{x}, \mathfrak{L}) \mid(\mathbf{x}, \mathfrak{L}) \in \mathcal{D}\left(J_{\eta, \lambda, \mu^{(1)}, \mu^{(2)}}^{\epsilon}\right)\right\}
$$

be the infimum of the functional $J_{\eta, \lambda, \mu^{(1)}, \mu^{(2)}}^{\epsilon}$. We now show that $\nu$ is a minimum, i.e., that $\nu$ is attained.

By definition of $\nu$ there exist $M>0$ and a sequence $\left\{\left(\mathbf{x}^{j}, \mathfrak{L}^{j}\right)\right\}_{j} \in$ $\mathcal{D}\left(J_{\eta, \lambda, \mu^{(1)}, \mu^{(2)}}^{\epsilon}\right)$ such that

$$
J_{\eta, \lambda, \mu^{(1)}, \mu^{(2)}}^{\epsilon}\left(\mathbf{x}^{j}, \mathfrak{L}^{j}\right) \rightarrow \nu \quad \text { and } \quad J_{\eta, \lambda, \mu^{(1)}, \mu^{(2)}}^{\epsilon} \leq M, \forall j .
$$

In particular, we have

$$
\mu^{(1)}\|\mathbf{x}\|^{2} \leq M \quad \text { and } \quad \mu^{(2)} \sum_{i}\left\|L_{i}\right\|^{2} \leq M .
$$

Thus,

$$
\|\mathbf{x}\| \leq\left(\frac{M}{\mu^{(1)}}\right)^{\frac{1}{2}} \text { and } \quad\left\|L_{i}\right\| \leq\left(\frac{M}{\mu^{(2)}}\right)^{\frac{1}{2}},
$$

which means that the sequences $\left\{\mathbf{x}^{j}\right\}_{j}$ and $\left\{\mathfrak{L}^{j}\right\}_{j}$ are uniformly bounded. Thus, there exist convergent subsequences of $\left\{\mathbf{x}^{j}\right\}_{j},\left\{\mathfrak{L}^{j}\right\}_{j}$ (for simplicity, again denoted by $\left\{\mathbf{x}^{j}\right\}_{j}$ and $\left\{\mathfrak{L}^{j}\right\}_{j}$ ) such that

$$
\mathbf{x}^{j} \rightarrow \overline{\mathbf{x}} \quad \text { and } \quad \mathfrak{L}^{j} \rightarrow \overline{\mathfrak{L}}
$$

for some $(\overline{\mathbf{x}}, \overline{\mathfrak{L}})$. In particular, we have that $\left(\mathbf{x}^{j}, \mathfrak{L}^{j}\right) \rightarrow(\overline{\mathbf{x}}, \overline{\mathfrak{L}})$. 

that

By the weakly lower semi-continuity of the functional $J_{\eta, \lambda, \mu^{(1)}, \mu^{(2)}}^{\epsilon}$, it holds

$$
\begin{aligned}
\nu & \leq J_{\eta, \lambda, \mu^{(1)}, \mu^{(2)}}^{\epsilon}(\overline{\mathbf{x}}, \overline{\mathfrak{L}}) \\
& \leq \liminf _{j} J_{\eta, \lambda, \mu^{(1)}, \mu^{(2)}}^{\epsilon}\left(\mathbf{x}^{j}, \mathfrak{L}^{j}\right) \\
& =\lim _{j} J_{\eta, \lambda, \mu^{(1)}, \mu^{(2)}}^{\epsilon}\left(\mathbf{x}^{j}, \mathfrak{L}^{j}\right) \\
& =\nu .
\end{aligned}
$$

Hence, $\nu=J_{\eta, \lambda, \mu^{(1)}, \mu^{(2)}}^{\epsilon}(\overline{\mathbf{x}}, \overline{\mathfrak{L}})$ is the minimum of the functional and $(\overline{\mathbf{x}}, \overline{\mathfrak{L}})$ is a global minimizer, i.e.,

$$
(\overline{\mathbf{x}}, \overline{\mathfrak{L}}) \in \arg \min _{(\mathbf{x}, \mathfrak{L})} J_{\eta, \lambda, \mu^{(1)}, \mu^{(2)}}^{\epsilon}(\mathbf{x}, \mathfrak{L})
$$

Which concludes the proof.

Now we prove the stability of the minima.

Theorem 2 (Stability) Let $\eta, \lambda, \mu^{(1)}, \mu^{(2)}>0$ be fixed parameters and let $\left\{\mathbf{y}^{\epsilon_{j}}\right\}_{j}$ be a sequence such that $\mathbf{y}^{\epsilon_{j}} \rightarrow \mathbf{y}^{\epsilon}$ as $j \rightarrow \infty$. Let $\left(\mathbf{x}^{j}, \mathfrak{L}^{j}\right)$ denote minimizers of $J_{\eta, \lambda, \mu^{(1)}, \mu^{(2)}}^{\epsilon_{j}}$ with data $\mathbf{y}^{\epsilon_{j}}$, then there exists a convergent subsequence of $\left\{\left(\mathbf{x}^{j}, \mathfrak{L}^{j}\right)\right\}_{j}$. Moreover, the limit of every convergent subsequence is a minimizer of the functional $J_{\eta, \lambda, \mu^{(1)}, \mu^{(2)}}^{\epsilon}$.

Proof According to the definition of $\left(\mathbf{x}^{j}, \mathfrak{L}^{j}\right)$ as minimizers of $J_{\eta, \lambda, \mu^{(1)}, \mu^{(2)}}^{\epsilon_{j}}$, we have that

$$
J_{\eta, \lambda, \mu^{(1)}, \mu^{(2)}}^{\epsilon_{j}}\left(\mathbf{x}^{j}, \mathfrak{L}^{j}\right) \leq J_{\eta, \lambda, \mu^{(1)}, \mu^{(2)}}^{\epsilon_{j}}(\mathbf{x}, \mathfrak{L}), \quad \forall(\mathbf{x}, \mathfrak{L}) \in \mathcal{D}\left(J_{\eta, \lambda, \mu^{(1)}, \mu^{(2)}}^{\epsilon}\right) .
$$

Let $(\tilde{\mathbf{x}}, \tilde{\mathfrak{L}}):=\left(\mathbf{x}_{\eta, \lambda, \mu^{(1)}, \mu^{(2)}}^{\epsilon}, \mathfrak{L}_{\eta, \lambda, \mu^{(1)}, \mu^{(2)}}^{\epsilon}\right)$ be a minimizer of $J_{\eta, \lambda, \mu^{(1)}, \mu^{(2)}}^{\epsilon}$. By the weakly lower semi-continuity, it holds that

$$
J_{\eta, \lambda, \mu^{(1)}, \mu^{(2)}}^{\epsilon_{j}}(\tilde{\mathbf{x}}, \tilde{\mathfrak{L}}) \rightarrow J_{\eta, \lambda, \mu^{(1)}, \mu^{(2)}}^{\epsilon}(\tilde{\mathbf{x}}, \tilde{\mathfrak{L}}) \text { as } j \rightarrow \infty
$$

Therefore, there exists a $\tilde{c}>0$ such that $J_{\eta, \lambda, \mu^{(1)}, \mu^{(2)}}^{\epsilon_{j}}(\tilde{\mathbf{x}}, \tilde{\mathfrak{L}}) \leq \tilde{c}$ for $j$ sufficiently large.

As in the proof of Theorem[1 we can conclude that the sequence $\left\{\left(\mathbf{x}^{j}, \mathfrak{L}^{j}\right)\right\}_{j}$ is uniformly bounded. Thus, there exists a convergent subsequence (for simplicity also denoted by $\left.\left\{\left(\mathbf{x}^{j}, \mathfrak{L}^{j}\right)\right\}_{j}\right)$ such that

$$
\mathbf{x}^{j} \rightarrow \overline{\mathbf{x}} \quad \text { and } \quad \mathfrak{L}^{j} \rightarrow \overline{\mathfrak{L}},
$$

for some $(\overline{\mathbf{x}}, \overline{\mathfrak{L}})$. 
In addition, (13) implies

$$
\begin{aligned}
J_{\eta, \lambda, \mu^{(1)}, \mu^{(2)}}^{\epsilon}\left(\overline{\mathbf{x}}, \overline{\mathfrak{L}}_{i}\right) & \leq \liminf _{j} J_{\eta, \lambda, \mu^{(1)}, \mu^{(2)}}^{\epsilon_{j}}\left(\mathbf{x}^{j}, \mathfrak{L}^{j}\right) \\
& \leq \limsup _{j} J_{\eta, \lambda, \mu^{(1)}, \mu^{(2)}}^{\epsilon_{j}}(\mathbf{x}, \mathfrak{L}) \\
& =\lim _{j} J_{\eta, \lambda, \mu^{(1)}, \mu^{(2)}}^{\epsilon_{j}}(\mathbf{x}, \mathfrak{L}) \\
& =J_{\eta, \lambda, \mu^{(1)}, \mu^{(2)}}^{\epsilon}(\mathbf{x}, \mathfrak{L})
\end{aligned}
$$

for all $(\mathbf{x}, \mathfrak{L}) \in \mathcal{D}\left(J_{\eta, \lambda, \mu^{(1)}, \mu^{(2)}}^{\epsilon}\right)$. In particular,

$$
J_{\eta, \lambda, \mu^{(1)}, \mu^{(2)}}^{\epsilon}(\overline{\mathbf{x}}, \overline{\mathfrak{L}}) \leq J_{\eta, \lambda, \mu^{(1)}, \mu^{(2)}}^{\epsilon}(\tilde{\mathbf{x}}, \tilde{\mathfrak{L}}) .
$$

Since $(\tilde{\mathbf{x}}, \tilde{\mathfrak{L}})$ is a minimizer of $J_{\eta, \lambda, \mu^{(1)}, \mu^{(2)}}^{\epsilon}$ by definition, we have that

$$
J_{\eta, \lambda, \mu^{(1)}, \mu^{(2)}}^{\epsilon}(\overline{\mathbf{x}}, \overline{\mathfrak{L}})=J_{\eta, \lambda, \mu^{(1)}, \mu^{(2)}}^{\epsilon}\left(\tilde{\mathbf{x}}, \tilde{\mathfrak{L}}_{i}\right)
$$

and thus

$$
\lim _{j \rightarrow \infty} J_{\eta, \lambda, \mu^{(1)}, \mu^{(2)}}^{\epsilon_{j}}\left(\mathbf{x}^{j}, \mathfrak{L}^{j}\right)=J_{\eta, \lambda, \mu^{(1)}, \mu^{(2)}}^{\epsilon}(\overline{\mathbf{x}}, \overline{\mathfrak{L}}),
$$

concluding the proof.

Definition 1 We call $\mathbf{x}^{\dagger}$ a minimum-norm solution of the noise-free version of (12) if

$$
\mathbf{x}^{\dagger}=\arg \min _{\mathbf{x}}\left\{\|\mathbf{x}\|^{2}+\sum_{i}\left\|P_{\mathbf{x}}^{(i)}\right\|^{2}: \mathbf{y}=\Phi \mathbf{x}\right\}
$$

We are now in the position to prove that (12) is a regularization method.

Theorem 3 (Regularization) Let $\left\{\mathbf{y}^{\epsilon_{j}}\right\}_{j}$ be a sequence of data such that $\left\|\mathbf{y}^{\epsilon_{j}}-\mathbf{y}\right\| \leq \epsilon_{j}$ with $\epsilon_{j} \rightarrow 0$. Assume that the regularization parameters $\eta_{j}=$ $\eta\left(\epsilon_{j}\right), \lambda_{j}=\lambda\left(\epsilon_{j}\right), \mu_{j}^{(1)}=\mu^{(1)}\left(\epsilon_{j}\right), \mu_{j}^{(2)}=\mu^{(2)}\left(\epsilon_{j}\right)$ fulfill $\eta_{j} \rightarrow \bar{\eta}(0<\bar{\eta}<\infty)$, $\lambda_{j} \rightarrow 0, \mu_{j}^{(1)} \rightarrow 0, \mu_{j}^{(2)} \rightarrow 0$ as well as

$$
\lim _{j \rightarrow \infty} \frac{\epsilon_{j}^{2}}{\mu_{j}^{(1)}}=0, \quad \lim _{j \rightarrow \infty} \frac{\mu_{j}^{(2)}}{\mu_{j}^{(1)}}=1 .
$$

Let $\left(\mathbf{x}^{j}, \mathfrak{L}^{j}\right):=\left(\mathbf{x}_{\eta, \lambda, \mu^{(1)}, \mu^{(2)}}^{\epsilon_{j}}, \mathfrak{L}_{i_{\eta, \lambda, \mu^{(1)}, \mu^{(2)}}^{\epsilon_{j}}}\right)$ be a minimizer of the functional $J_{\eta_{j}, \lambda_{j}, \mu_{j}^{(1)}, \mu_{j}^{(2)}}^{\epsilon_{j}}$ obtained from the noisy data $\mathbf{y}_{\epsilon_{j}}$, regularization parameters $\eta_{j}$, $\lambda_{j}, \mu_{j}^{(1)}, \mu_{j}^{(2)}$.

Then there exists a convergent subsequence of $\left\{\left(\mathbf{x}^{j}, \mathfrak{L}^{j}\right)\right\}_{j}$, each limit point $\left(\overline{\mathbf{x}}^{j}, \overline{\mathfrak{L}}^{j}\right)$ is such that $\bar{L}_{i}^{j}=P_{\mathbf{x}^{\dagger}}^{(i)}$, and $\overline{\mathbf{x}}$ is a minimum-norm solution of $\mathbf{y}=\Phi \mathbf{x}$, i.e., $\overline{\mathbf{x}}=\mathbf{x}^{\dagger}$. 
Proof The minimizing property of $\left(\mathbf{x}^{j}, \mathfrak{L}^{j}\right)$ implies that

$$
J_{\eta_{j}, \lambda_{j}, \mu_{j}^{(1)}, \mu_{j}^{(2)}}^{\epsilon_{j}}\left(\mathbf{x}^{j}, \mathfrak{L}^{j}\right) \leq J_{\eta_{j}, \lambda_{j}, \mu_{j}^{(1)}, \mu_{j}^{(2)}}^{\epsilon_{j}}(\mathbf{x}, \mathfrak{L}), \quad \forall(\mathbf{x}, \mathfrak{L}) \in \mathcal{D}\left(J_{\eta, \lambda, \mu^{(1)}, \mu^{(2)}}^{\epsilon}\right)
$$

In particular, denoting by $\mathbf{0}$ the $\mathfrak{L}$ where $L_{i}=0$ for all $i$, we obtain

$$
\begin{aligned}
0 & \leq J_{\eta_{j}, \lambda_{j}, \mu_{j}^{(1)}, \mu_{j}^{(2)}}^{\epsilon_{j}}\left(\mathbf{x}^{j}, \mathfrak{L}^{j}\right) \\
& \leq J_{\eta_{j}, \lambda_{j}, \mu_{j}^{(1)}, \mu_{j}^{(2)}}^{\epsilon_{j}}\left(\mathbf{x}^{\dagger}, \mathbf{0}\right) \\
& =\left\|\mathbf{y}^{\epsilon_{j}}-\Phi \mathbf{x}^{\dagger}\right\|^{2}+\mu_{j}^{(1)}\left\|\mathbf{x}^{\dagger}\right\|^{2}+\mu_{j}^{(2)} \sum_{i}\left\|P_{\mathbf{x}^{\dagger}}^{(i)}\right\|^{2} \\
& \leq \epsilon_{j}^{2}+\mu_{j}^{(1)}\left\|\mathbf{x}^{\dagger}\right\|^{2}+\mu_{j}^{(2)} \sum_{i}\left\|P_{\mathbf{x}^{\dagger}}^{(i)}\right\|^{2}
\end{aligned}
$$

where $\mathbf{x}^{\dagger}$ denotes a minimum-norm solution of the equation $\mathbf{y}=\Phi \mathbf{x}$ according to Definition 1

Combining this estimate with the assumption on the regularization parameters, we conclude that the sequences $\left\|\mathbf{y}_{\epsilon_{j}}-\Phi \mathbf{x}^{j}\right\|^{2}, G_{\delta}\left(P_{\mathbf{x}^{j}}^{(i)}\right),\left\|\mathbf{x}^{j}\right\|^{2}$ and $\left\|P_{\mathbf{x}^{j}}^{(i)}\right\|^{2}$ are uniformly bounded. Thus there exists a convergent subsequence $\left\{\left(\mathbf{x}^{m}, L_{i}^{m}\right)\right\}_{m}:=\left\{\left(\mathbf{x}^{j_{m}}, L_{i}^{j_{m}}\right)\right\}_{j_{m}}$ of $\left\{\left(\mathbf{x}^{j}, L_{i}^{j}\right)\right\}_{j}$ such that

$$
\left(\mathbf{x}^{m}, \mathfrak{L}^{m}\right) \rightarrow(\overline{\mathbf{x}}, \overline{\mathfrak{L}}) \text { as } m \rightarrow \infty .
$$

We will now prove that for the limit $(\overline{\mathbf{x}}, \overline{\mathfrak{L}})$ it holds that $\overline{\mathfrak{L}}_{i}=P_{\overline{\mathbf{x}}}^{(i)}$ and that $\overline{\mathbf{x}}$ is a minimum-solution.

From the continuity of the norm we derive

$$
\begin{aligned}
0 & \leq\|\mathbf{y}-\Phi \overline{\mathbf{x}}\|^{2}+\bar{\eta} \sum_{i}\left\|P_{\overline{\mathbf{x}}}^{(i)}-\bar{L}_{i}\right\|^{2} \\
& \leq \liminf _{m \rightarrow \infty}\left\{\left\|\mathbf{y}-\Phi \mathbf{x}^{m}\right\|^{2}+\eta_{m} \sum_{i}\left\|P_{\mathbf{x}^{m}}^{(i)}-L_{i}^{m}\right\|^{2}\right\} \\
& \stackrel{(a)}{\leq} \liminf _{m \rightarrow \infty}\left\{\epsilon_{m}^{2}+\mu_{m}^{(1)}\left\|\mathbf{x}^{\dagger}\right\|^{2}+\mu_{m}^{(2)} \sum_{i}\left\|P_{\mathbf{x}^{\dagger}}^{(i)}\right\|^{2}\right\} \\
& \stackrel{(b)}{=} 0
\end{aligned}
$$

where $(a)$ holds thanks to (15) and (b) follows from the assumptions on the regularization parameters. Then we have that $\mathbf{y}=\Phi \overline{\mathbf{x}}$ and $\bar{L}_{i}=P_{\overline{\mathbf{x}}}^{(i)}$. 
From inequality (15), we know that

$$
\begin{aligned}
\left\|\mathbf{x}^{m}\right\|^{2}+\frac{\mu_{m}^{(2)}}{\mu_{m}^{(1)}} \sum_{i}\left\|P_{\mathbf{x}^{m}}^{(i)}\right\|^{2} & \leq \frac{1}{\mu_{m}^{(1)}} J_{\eta_{m}, \lambda_{m}, \mu_{m}^{(1)}, \mu_{m}^{(2)}}^{\epsilon_{m}}\left(\mathbf{x}^{m}, \mathfrak{L}^{m}\right) \\
& \leq \frac{\epsilon_{m}^{2}}{\mu_{m}^{(1)}}+\left\|\mathbf{x}^{\dagger}\right\|^{2}+\frac{\mu_{m}^{(2)}}{\mu_{m}^{(1)}} \sum_{i}\left\|P_{\mathbf{x}^{\dagger}}^{(i)}\right\|^{2} .
\end{aligned}
$$

The continuity of the norm results in

$$
\begin{aligned}
\|\overline{\mathbf{x}}\|^{2}+\sum_{i}\left\|P_{\overline{\mathbf{x}}}^{(i)}\right\|^{2} & =\|\overline{\mathbf{x}}\|^{2}+\sum_{i}\left\|\bar{L}_{i}\right\|^{2} \\
& =\liminf _{m \rightarrow \infty}\left\{\left\|\mathbf{x}^{m}\right\|^{2}+\sum_{i}\left\|L_{i}^{m}\right\|^{2}\right\} \\
& =\liminf _{m \rightarrow \infty}\left\{\left\|\mathbf{x}^{m}\right\|^{2}+\frac{\mu_{m}^{(2)}}{\mu_{m}^{(1)}} \sum_{i}\left\|L_{i}^{m}\right\|^{2}\right\} \\
& \leq \liminf _{m \rightarrow \infty}\left\{\frac{\epsilon_{m}^{2}}{\mu_{m}^{(1)}}+\left\|\mathbf{x}^{\dagger}\right\|^{2}+\frac{\mu_{m}^{(2)}}{\mu_{m}^{(1)}} \sum_{i}\left\|P_{\mathbf{x}^{\dagger}}^{(i)}\right\|^{2}\right\} \\
& =\left\|\mathbf{x}^{\dagger}\right\|^{2}+\sum_{i}\left\|P_{\mathbf{x}^{\dagger}}^{(i)}\right\|^{2},
\end{aligned}
$$

where the last steps hold according to (15) and (14), respectively. Therefore,

$$
\|\overline{\mathbf{x}}\|^{2}+\sum_{i}\left\|P_{\overline{\mathbf{x}}}^{(i)}\right\|^{2}=\left\|\mathbf{x}^{\dagger}\right\|^{2}+\sum_{i}\left\|P_{\mathbf{x}^{\dagger}}^{(i)}\right\|^{2},
$$

i.e., $\overline{\mathbf{x}}=\mathbf{x}^{\dagger}$.

As $\bar{L}_{i}=P_{\overline{\mathbf{x}}}^{(i)}$ we conclude that $\overline{\mathbf{x}}$ is a minimum-norm solution, which concludes the proof.

\section{Algorithm}

For computing a stationary point of the functional $J_{\eta, \lambda, \mu^{(1)}, \mu^{(2)}}^{\epsilon}$ defined above, we would like to formulate an alternating descent algorithm. However, being the functional non-continuous (and thus non-differentiable), it is hard to define a convergent numerical method. To this end, we slightly modify the functional $J_{\eta, \lambda, \mu^{(1)}, \mu^{(2)}}^{\epsilon}$ to make this approach feasible.

The discontinuities of the functional $J_{\eta, \lambda, \mu^{(1)}, \mu^{(2)}}^{\epsilon}$ appear as jumps in the construction of $P_{\mathbf{x}}^{(i)}$ thus it is possible to join the jump with a differentiable joint. We do not describe in details here this approach since it is technical and does not add anything of interest to our analysis. For the following of this section, with abuse of notation, we will denote again by $J_{\eta, \lambda, \mu^{(1)}, \mu^{(2)}}^{\epsilon}$ the 
smoothed functional and describe an algorithm to obtain one of its critical points.

Since we want to employ an alternating descent technique, we decouple the functional $J_{\eta, \lambda, \mu^{(1)}, \mu^{(2)}}^{\epsilon}$ with respect to the variables $\mathbf{x}$ and $L_{i}$ as follows

- The $L_{i}$-subproblems, for $i=1 \ldots \ell$, are

$$
\hat{L}_{i}=\arg \min _{L_{i}} \eta\left\|P_{\mathbf{x}}^{(i)}-L_{i}\right\|^{2}+\eta \lambda G_{\delta}\left(L_{i}\right)+\mu^{(2)}\left\|L_{i}\right\|^{2} .
$$

- The $\mathbf{x}$-subproblem is

$$
\hat{\mathbf{x}}=\arg \min _{\mathbf{x}}\|\mathbf{y}-\Phi \mathbf{x}\|^{2}+\eta \sum_{i}\left\|P_{\mathbf{x}}^{(i)}-L_{i}\right\|^{2}+\mu^{(1)}\|\mathbf{x}\|^{2} .
$$

For the proof of convergence we follow 21].

We first consider (16). Due to the smoothness of the norm and of the SRF, we can tackle the $L_{i}$-subproblems by the gradient descent method.

Let us denote by $f_{i}\left(L_{i}\right)$ the following

$$
f_{i}\left(L_{i}\right)=\eta\left\|P_{\mathbf{x}}^{(i)}-L_{i}\right\|^{2}+\eta \lambda G_{\delta}\left(L_{i}\right)+\mu^{(2)} \sum_{i}\left\|L_{i}\right\|^{2} .
$$

In order to apply the gradient descent method we first have to compute $\nabla f$, namely,

$$
\nabla f_{i}\left(L_{i}\right)=-2 \eta\left(P_{\mathbf{x}}^{(i)}-L_{i}\right)+\eta \lambda \nabla G_{\delta}\left(L_{i}\right)+2 \mu^{(2)} L_{i} .
$$

We can compute $\nabla G_{\delta}\left(L_{i}\right)$ as in [19. Consider the singular values decomposition of $L_{i}$, we recall that $L_{i} \in \mathbb{R}^{n \times m}$, given by

$$
L_{i}=U \Sigma V^{T},
$$

where $U \in \mathbb{R}^{n \times n}$ and $V \in \mathbb{R}^{m \times m}$ are orthogonal matrices and $\Sigma \in \mathbb{R}^{n \times m}$ is a diagonal matrix whose diagonal nonzero entries are $\sigma_{1} \geq \sigma_{2} \geq \cdots \geq \sigma_{r} \geq 0$, with $r=\min \{n, m\}$. Thus the gradient of the $\mathrm{SRF}$ is

$$
\nabla G_{\delta}\left(L_{i}\right)=-U \operatorname{diag}\left(-\frac{\sigma_{1}}{\delta^{2}} \mathrm{e}^{-\sigma_{1}^{2} / 2 \delta^{2}}, \cdots,-\frac{\sigma_{\mathrm{r}}}{\delta^{2}} \mathrm{e}^{-\sigma_{\mathrm{r}}^{2} / 2 \delta^{2}}\right) V^{T},
$$

where diag $\left(\lambda_{1}, \ldots, \lambda_{r}\right)$ denotes the $n \times m$ diagonal matrix whose the first $r$ diagonal entries are $\lambda_{1}, \ldots, \lambda_{r}$ and the remaining are zero.

The $k$ th iteration of the gradient descent method applied to $f$ has the following form

$$
L_{i}^{(k+1)}=L_{i}^{(k)}-\tau^{(k)} \nabla f_{i}\left(L_{i}^{(k)}\right),
$$

where $\tau^{(k)}>0$ is the step-length at step $k$. For the convergence we need to select the appropriate $\tau^{(k)}$, in particular we use the Armijo rule described in Algorithm 1] see, e.g., [21]. 
Algorithm 1 (Armijo rule choice) Let $g(\mathbf{z})$ be a continuously differentiable function and let $\beta, \gamma \in(0,1)$ be fixed parameters. The Armijo steplength $\tau$ is obtained as

$$
\begin{aligned}
& \text { while } g(\mathbf{z}-\tau \nabla g(\mathbf{z}))>g(\mathbf{z})-\beta \tau\|\nabla g(\mathbf{z})\|^{2} \text { do } \\
& \qquad \begin{array}{l}
\mid \\
\text { end }
\end{array}
\end{aligned}
$$

The well posedness of Algorithm [1 is proven, e.g., in [21, Proposition 4.1].

We move to the $\mathbf{x}$-subproblem. Being the problem nonlinear, due to the presence of $P_{\mathbf{x}}^{(i)}$, but smooth (thanks to the smoothed technique described at the beginning of this section), we can obtain a local minimum of (17) by means of the nonlinear conjugate gradient (CG) method, see, e.g., [15].

In Algorithm 2 we summarize our proposal.

Algorithm 2 (Theoretical Proposal) Consider the (smoothed version of the) functional $J_{\eta, \lambda, \mu^{(1)}, \mu^{(2)}}^{\epsilon}$ defined in (12). Let $\mathbf{x}^{(0)}$ be an initial guess for $\mathbf{x}$.

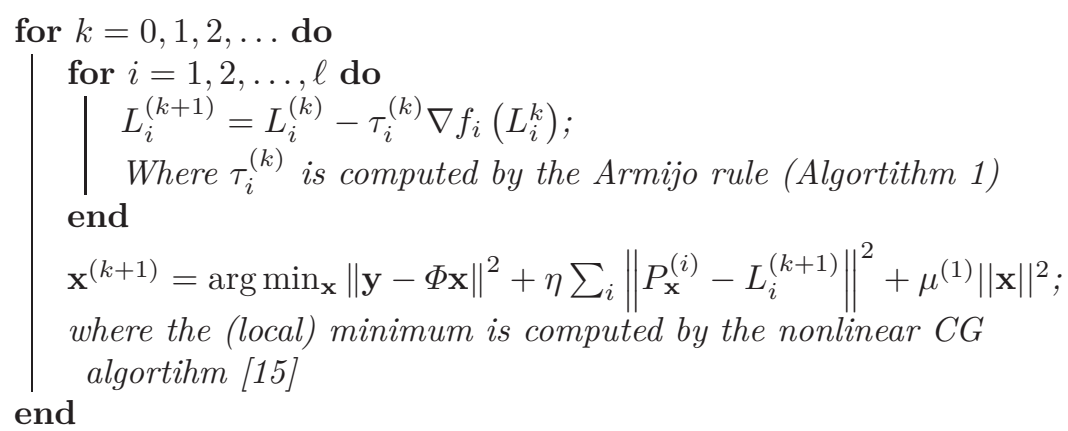

Let us call $T_{L_{i}}$ and $T_{\mathbf{x}}$ the update rules for $L_{i}$ and $\mathbf{x}$, respectively, then it holds

Proposition 1 The update rules $T_{L_{i}}$ and $T_{\mathbf{x}}$ defined in Algorithm Q satisfy the following properties

(i) Let $\left\{\mathbf{x}^{(k)}, L_{i}^{(k)}\right\}$ be a sequence, we have that

$$
J_{\eta, \lambda, \mu^{(1)}, \mu^{(2)}}^{\epsilon}\left(\mathbf{x}^{(k)}, T_{L_{i}}\left(L_{i}^{(k)}\right)\right) \leq J_{\eta, \lambda, \mu^{(1)}, \mu^{(2)}}^{\epsilon}\left(\mathbf{x}^{(k)}, L_{i}^{(k)}\right)
$$

and

$$
J_{\eta, \lambda, \mu^{(1)}, \mu^{(2)}}^{\epsilon}\left(T_{\mathbf{x}}\left(\mathbf{x}^{(k)}\right), L_{i}^{(k)}\right) \leq J_{\eta, \lambda, \mu^{(1)}, \mu^{(2)}}^{\epsilon}\left(\mathbf{x}^{(k)}, L_{i}^{(k)}\right),
$$

for all $k$ and for all $i$.

(ii) Let $\left\{\mathbf{x}^{(k)}, L_{i}^{(k)}\right\}$ be a converging sequence to some $\left(\overline{\mathbf{x}}, \bar{L}_{i}\right)$.

If

$$
\lim _{k \rightarrow \infty} J_{\eta, \lambda, \mu^{(1)}, \mu^{(2)}}^{\epsilon}\left(\mathbf{x}^{(k)}, L_{i}^{(k)}\right)-J_{\eta, \lambda, \mu^{(1)}, \mu^{(2)}}^{\epsilon}\left(\mathbf{x}^{(k)}, T_{L_{i}}\left(L_{i}^{(k)}\right)\right)=0,
$$

then we have that

$$
\nabla_{L_{i}} J_{\eta, \lambda, \mu^{(1)}, \mu^{(2)}}^{\epsilon}\left(\overline{\mathbf{x}}, \bar{L}_{i}\right)=\mathbf{0}
$$


If

$$
\lim _{k \rightarrow \infty} J_{\eta, \lambda, \mu^{(1)}, \mu^{(2)}}^{\epsilon}\left(\mathbf{x}^{(k)}, L_{i}^{(k)}\right)-J_{\eta, \lambda, \mu^{(1)}, \mu^{(2)}}^{\epsilon}\left(T_{\mathbf{x}}\left(\mathbf{x}^{(k)}\right), L_{i}^{(k)}\right)=0,
$$

then we have that

$$
\nabla_{\mathbf{x}} J_{\eta, \lambda, \mu^{(1)}, \mu^{(2)}}^{\epsilon}\left(\overline{\mathbf{x}}, \bar{L}_{i}\right)=\mathbf{0}
$$

(iii) Let $\left\{\mathrm{x}^{(k)}, L_{i}^{(k)}\right\}$ be a converging sequence to some $\left(\overline{\mathbf{x}}^{(k)}, \bar{L}_{i}^{(k)}\right)$.

If

$$
\lim _{k \rightarrow \infty} J_{\eta, \lambda, \mu^{(1)}, \mu^{(2)}}^{\epsilon}\left(\mathbf{x}^{(k)}, L_{i}^{(k)}\right)-J_{\eta, \lambda, \mu^{(1)}, \mu^{(2)}}^{\epsilon}\left(\mathbf{x}^{(k)}, T_{L_{i}}\left(L_{i}^{(k)}\right)\right)=0,
$$

then we have that

$$
\lim _{k \rightarrow \infty}\left\|T_{L_{i}}\left(L_{i}^{(k)}\right)-L_{i}^{(k)}\right\|=0 .
$$

If

$$
\lim _{k \rightarrow \infty} J_{\eta, \lambda, \mu^{(1)}, \mu^{(2)}}^{\epsilon}\left(\mathbf{x}^{(k)}, L_{i}^{(k)}\right)-J_{\eta, \lambda, \mu^{(1)}, \mu^{(2)}}^{\epsilon}\left(T_{\mathbf{x}}\left(\mathbf{x}^{(k)}\right), L_{i}^{(k)}\right)=0
$$

then we have that

$$
\lim _{k \rightarrow \infty}\left\|T_{\mathbf{x}}\left(\mathbf{x}^{(k)}\right)-\mathbf{x}^{(k)}\right\|=0
$$

Proof The proofs of these properties for $T_{L_{i}}$ can be found in [21, Section 4], while the properties of $T_{\mathbf{x}}$ are shown in [21, Section 5].

We can now state our main result

Theorem 4 Let $\left\{\mathbf{x}^{(k)}, L_{i}^{(k)}\right\}$ denotes the iterates generated by Algorithm $\mathbf{Q}$, then every limit point of $\left\{\mathbf{x}^{(k)}, L_{i}^{(k)}\right\}$ is a stationary point of $J_{\eta, \lambda, \mu^{(1)}, \mu^{(2)}}^{\epsilon}$ and

$$
\lim _{k \rightarrow \infty} \nabla J_{\eta, \lambda, \mu^{(1)}, \mu^{(2)}}^{\epsilon}\left(\mathbf{x}^{(k)}, L_{i}^{(k)}\right)=\mathbf{0} .
$$

Proof We omit the proof since it can be copied from the proof of 21, Theorem 3.3], when is noted that the level set $\mathcal{L}=\left\{\left(\mathbf{x}, L_{i}\right): J_{\eta, \lambda, \mu^{(1)}, \mu^{(2)}}^{\epsilon}\left(\mathbf{x}, L_{i}\right) \leq\right.$ $\left.J_{\eta, \lambda, \mu^{(1)}, \mu^{(2)}}^{\epsilon}\left(\mathbf{x}^{(0)}, L_{i}^{(0)}\right)\right\}$ is compact since $J_{\eta, \lambda, \mu^{(1)}, \mu^{(2)}}^{\epsilon}$ is coercive. 


\subsection{Implementation of Algorithm 2}

The implementation of Algorithm 2 is non-trivial and it could be computationally demanding. Hence we propose here an algorithm which approximate Algorithm 2 and that will be used in the numerical experiments. We would like to stress that, even though the two algorithms are going to be similar, the proof of Theorem 4 cannot be applied to the new variant of the algorithm.

The main problem in Algorithm 2 is the computation of $\mathbf{x}^{(k+1)}$. To avoid the computation of the smooth joint of the jumps in $J_{\eta, \lambda, \mu^{(1)}, \mu^{(2)}}^{\epsilon}$ and to avoid the usage of the nonlinear CG method we proceed as follows.

Consider $P_{\mathbf{x}}^{(i)}$, we can write it as

$$
P_{\mathbf{x}}^{(i)}=P^{(i)}(\mathbf{x}) \mathbf{x}
$$

where $P^{(i)}(\mathbf{x})$ is an appropriate diagonal matrix which depends, in a non-linear way, on $\mathbf{x}$, see (11). Observe that, by definition of $P^{(i)}(\mathbf{x})$, it holds

$$
\sum_{i}\left(P^{(i)}(\mathbf{x})\right)^{t} P^{(i)}(\mathbf{x})=I, \quad \forall \mathbf{x}
$$

where $I$ denotes the identity matrix.

At the $k$ th step we make the following approximation

$$
P^{(i)}(\mathbf{x}) \approx P^{(i)}\left(\mathbf{x}^{(k)}\right), \quad \forall \mathbf{x},
$$

in this way we have removed the dependency from $\mathbf{x}$ in the construction of $P^{(i)}(\mathbf{x})$. Intuitively this approximation can be seen as a Taylor expansion of order zero of $P^{(i)}(\mathbf{x})$ in $\mathbf{x}=\mathbf{x}^{(k)}$. Then we have

$$
\mathbf{x}^{(k+1)} \approx \arg \min _{\mathbf{x}}\|\mathbf{y}-\Phi \mathbf{x}\|^{2}+\eta \sum_{i}\left\|P^{(i)}\left(\mathbf{x}^{(k)}\right) \mathbf{x}-L_{i}^{k+1}\right\|^{2}+\mu^{(1)}\|\mathbf{x}\|^{2}
$$

The solution of (19) $\mathbf{x}^{(k+1)}$ can be computed by solving the associated normal equations

$$
\begin{aligned}
& \left(\Phi^{*} \Phi+\eta \sum_{i}\left(P^{(i)}\left(\mathbf{x}^{(\mathbf{k})}\right)\right)^{t} P^{(i)}\left(\mathbf{x}^{(\mathbf{k})}\right)+\mu^{(1)} I\right) \mathbf{x}^{(k+1)} \\
& =\Phi^{*} \mathbf{y}+\eta \sum_{i} P^{(i)}\left(\mathbf{x}^{(k)}\right) L_{i}^{(k+1)}
\end{aligned}
$$

In force of (18) the normal equations (20) can be rewritten as

$$
\left(\Phi^{*} \Phi+\left(\eta+\mu^{(1)}\right) I\right) \mathbf{x}^{(k+1)}=\Phi^{*} \mathbf{y}+\eta \sum_{i} P^{(i)}\left(\mathbf{x}^{(k)}\right) L_{i}^{(k+1)} .
$$

We summarize our algorithmic proposal in the following 
Algorithm 3 (Our Proposal) Consider the functional $J_{\eta, \lambda, \mu^{(1)}, \mu^{(2)}}^{\epsilon}$ defined in (12). Let $\mathbf{x}^{(0)}$ be an initial guess for $\mathbf{x}$.

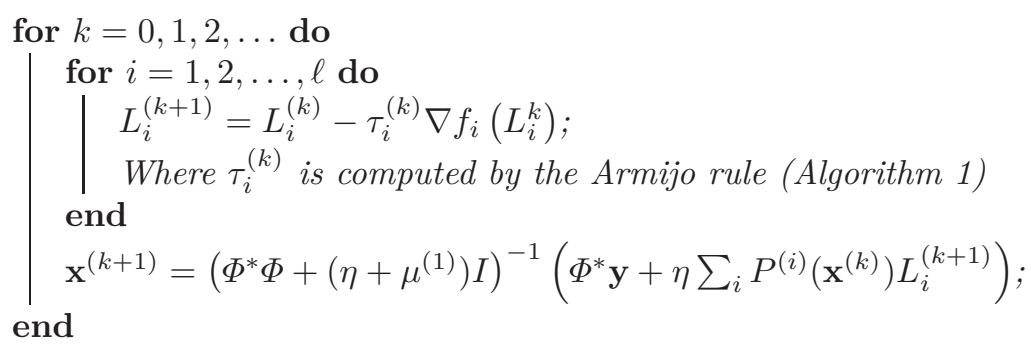

In the framework of CS, a possible choice for $\Phi$ is $\Phi=D F$, where $F$ is the Fourier matrix and $D$ is the downsampling operator that selects only few rows of $F$. When $\Phi$ has this structure then $\mathbf{x}^{(k+1)}$ in Algorithm 3 can be easily computed by

$$
\mathbf{x}^{(k+1)}=F^{*}\left(\left(D^{t} D+\left(\eta+\mu^{(1)}\right) I\right)^{-1}\left(D \mathbf{y}+\eta F \sum_{i} P^{(i)}\left(\mathbf{x}^{(k)}\right) L_{i}^{(k+1)}\right) .\right.
$$

Thus computing $\mathbf{x}^{(k+1)}$ requires the solution of a diagonal system and two applications of the Fourier matrix, which can be done quickly by means of the fft algorithm.

\section{Numerical experiments}

In this section, we present some numerical results for CS image recovery. We compare the proposed method with several state-of-the-art approaches for CS image recovery, namely

- The tvqc-newton method [9] is one of the $\ell_{1}$-magic method that reconstructs sparse signals by a convex program by solving the minimization problem

$$
\min T V(\mathbf{x}) \quad \text { s.t. } \quad\|\Phi \mathbf{x}-\mathbf{y}\|_{2} \leq \epsilon
$$

where $T V(\mathbf{x})$ denotes the total variation (TV) of $\mathbf{x}$ and $\epsilon$ is the norm of the noise in observation. The tvqc-newton method adopts Newton iteration for solving (21). All parameters are set to default values or automatically chosen as described in [9. This method exploits the sparsity of the gradients.

- The NESTA method [4] (short for Nesterov's algorithm) solves a smoothed version of (21), i.e.,

$$
\min T V_{\mu}(\mathbf{x}) \quad \text { s.t. } \quad\|\Phi \mathbf{x}-\mathbf{y}\|_{2} \leq \epsilon .
$$

Where $T V_{\mu}(\mathbf{x})$ is the smoothed version of the TV norm defined in 4. The parameter $\mu$ is crucial for this algorithm, in fact it affects both the accuracy and the speed of convergence. As suggested in the paper we set $\mu=0.2$. 
- The generalized higher degree total variation regularization method (generalized HDTV) 24 extends the second degree HDTV to the third degree HDTV, and significantly preserves and enhances features of the images such as edges or ridges. In particular, this method can be applied to compressive sensing, denoising, and deconvolution of three-dimensional datasets. This generalization is obtained by considering the gradients not only in the $x$ and $y$ directions, but in many possible directions simultaneously.

- The NLR-CS-baseline method and the NLR-CS method [16] transform the self-similarity of an image into low-rank prior by performing block matching technology [14. The rank minimization problem is NP-hard, thus the authors consider the nuclear norm and the log det function as relaxation of the rank. In the next experiments, the parameters are set to their default values.

- The SRF-CS method [19] adopts the non-convex and smooth function (5) to obtain a more accurate surrogate for the rank, with respect to the nuclear norm and the log det function. All the parameters are set to default except that the iteration number. The iteration number is the same as the one of the proposed algorithm in the next experiments.

When not stated otherwise, the parameters involved in the considered methods are fine tuned by trial and error.

The CS measurements are generated by random sampling or pseudo-radial sampling the Fourier coefficients of test images. The number of measurements is $M=r N$, where $r$ is the sampling rate. The accuracy of the solution is measured by the peak signal to noise ratio (PSNR) and Structural SIMilarity (SSIM) [32. The PSNR is defined as

$$
\operatorname{PSNR}=20 \log _{10} \frac{\max _{\mathrm{f}} \cdot N}{\|\mathbf{f}-\mathbf{g}\|}
$$

where $\mathbf{f}$ and $\mathbf{g}$ are the ground truth image and the restored image respectively, and $\max _{\mathrm{f}}$ is the maximum pixel value of image $\mathbf{f}$. The PSNR and SSIM respectively evaluate the similarity between the ground truth image and the restored image based on the mean squared error and the structural information. The structural information is based on the fact that the pixels have strong inter-dependencies especially when they are spatially close. These dependencies carry important structural information of the objects in the visual scene. The SSIM has been proven to be consistent with human visual perception.

The main parameters of the proposed algorithm are set as follows: patch size $\sqrt{n}=6$; the number of similar patches for each exemplary patch $m=45$; initialized value $\delta^{(0)}=\bar{\delta}$, where $\bar{\delta}$ is a constant around three times of the largest singular value of initial $L_{i}$ [19; the fixed parameters $\mu_{1}=\mu_{2}=0.0001$ and $\eta=0.05$; the parameter $\lambda$ is chosen by trial and error like the regularization parameter of the compared methods; the iteration number is $S=100$. To achieve better performance, we select exemplary patch in each 5 pixels along both horizontal and vertical directions. 
Number Our first example is the Number image with size $256 \times 256$. The observed image is generated by adding Gaussian white noise with 0.15 standard deviation. We reconstruct the Number image from $M=0.05 \mathrm{~N}$ measurements by randomly sampling its Fourier transform coefficients. From the visual inspection of the reconstructed images in Figure 1(b) and (c) we can see that the restorations obtained with the tvqc-newton and NESTA methods contain a lot artifacts and noise. The NLR-CS-baseline and NLR-CS methods, shown in Figure 1(d) and (i) are not able to precisely reconstruct the edges, resulting in "blurred" reconstructions. We clearly see from the visual inspection of Figure 1(j) that the restoration obtained with the generalized HDTV method contains many noise artifacts and ringing effects. However, the SRF-CS approach and the proposed method achieve high quality restored images, which attain sharp edges and reconstruct clearer images than the other compared methods, see Figure 1(k) and (l).

In Table 1 we show the PSNR and SSIM values for each reconstruction.

We would like to stress that our proposal provides a slightly lower PSNR value with respect to the SRF-CS method, but achieves a higher SSIM value.

Moon We now consider the Moon image $(256 \times 256$ pixels). In Figure 2 we show the restored results of the considered methods. The observed image is corrupted by Gaussian white noise with 0.2 standard deviation. We recover the corrupted image from $M=0.15 N$ measurements by randomly sampling its Fourier transform coefficients. From the PSNR and SSIM values in Table 1 we can observe that the NLR-CS-baseline method obtains better performance than the tvqc-newton, NESTA, generalized HDTV, and NLR-CS methods, which is confirmed by the visual inspection of the reconstructions. However, the proposed method recovers more details and obtains a much clearer image than NLR-CS-baseline and SRF-CS methods. In the close-ups of the restored images, we can see that smallest protruding points are better reconstructed by Algorithm 3 than by the SRF-CS method.

Head $\mathcal{E}^{3}$ Einstein In these two examples we consider the Head and Einstein images $(256 \times 256$ pixels $)$ with different random sampling rates and noise levels, see Figure 3 and 4 . The Head image is reconstructed from $M=0.1 N$ measurements corrupted by Gaussian white noise with 0.1 standard deviation, while the Einstein image is restored from $M=0.2 N$ measurements affected by Gaussian white noise with 0.3 standard deviation. In both cases we can observe that the restored Head and Einstein images obtained by tvqc-newton and NESTA methods are not accurate, as illustrated in Figure 3(b) and (f) and Figure 4(b) and (f). The generalized HDTV approach creates some staircase effect in Figure 3(c) and Figure 4(c). The proposed method and the SRF-CS method achieve clearer images when compared with the other methods even for large noise levels. Thus, we can infer that the proposed method is robust. In addition, the PSNR and SSIM values of the proposed method are very similar to the ones obtained with the SRF-CS approach, as shown in Table 1 . 


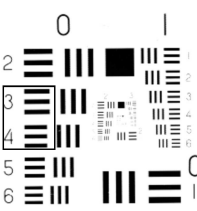

(a)

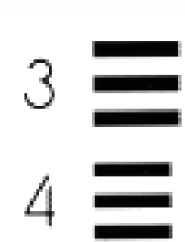

(e)

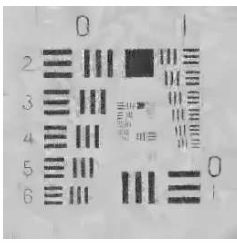

(i)

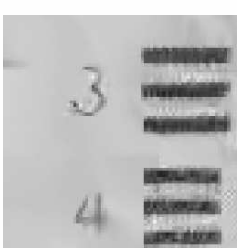

(m)

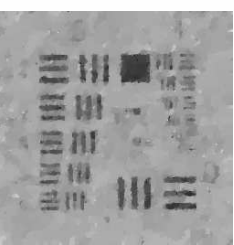

(b)

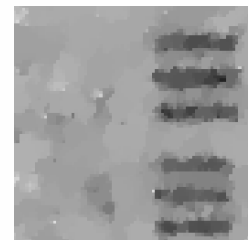

(f)

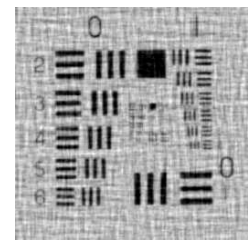

(j)

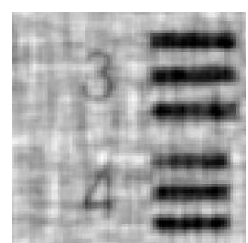

(n)

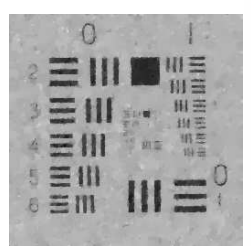

(c)

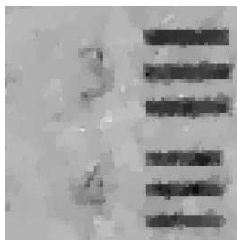

(g)

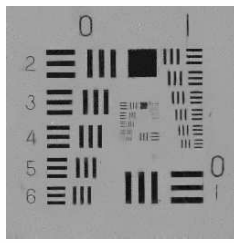

(k)

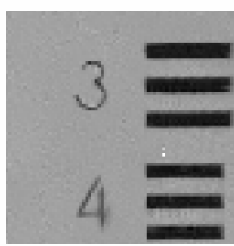

(o)

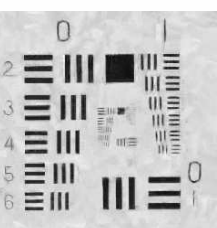

(d)

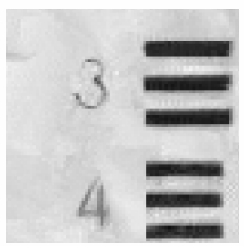

(h)

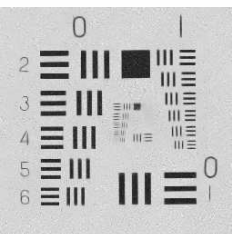

(1)

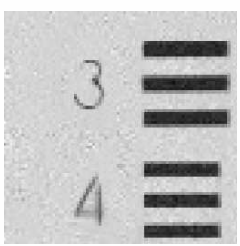

(p)

Fig. 1 Restored Number images from $0.05 \mathrm{~N}$ measurements with 0.15 Gaussian noise. (a/e) true image/Zoom (b/f) tvqc-newton/Zoom, (c/g) NESTA/Zoom, (d/h) NLRCS-baseline/Zoom, (i/m) NLR-CS/Zoom, (j/n) generalized HDTV/Zoom, (k/o) SRFCS/Zoom, (l/p) Algorithm 3 /Zoom.

To better show the differences in quality of the restorations, Figure 5 reports the residual images. We define the residual images as $|\mathbf{f}-\mathbf{g}|$, where $\mathbf{f}$ is the true image, $\mathbf{g}$ is the restored image and the absolute value is computed component wise. For the comparison to be fair, we scale all the residual image between the minimum and the maximum value attained by all residual images. In Figure 3(b) and (f) and Figure 4(b) and (f), the lower quality of the restored images obtained by tvqc-newton method and NESTA method is evident. Hence, we compare only generalized HDTV, NLR-CS-baseline, NLRCS and SRF-CS methods with the proposed approach in Figure 5. By visual 


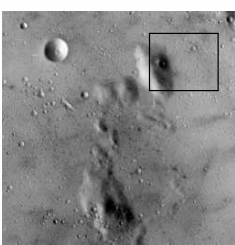

(a)

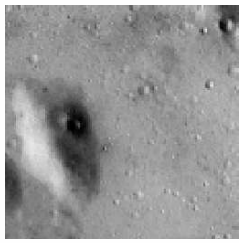

(e)

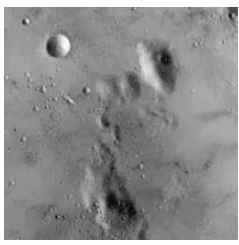

(i)

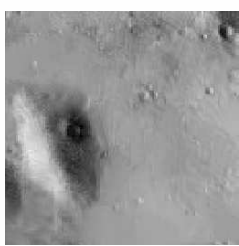

(m)

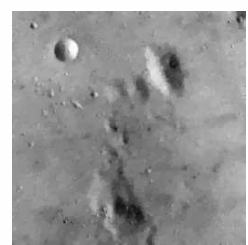

(b)

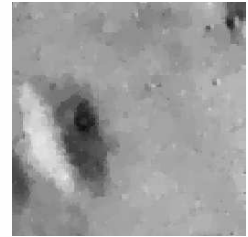

(f)

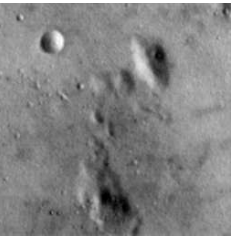

(j)

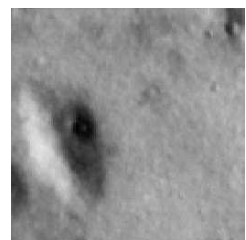

(n)

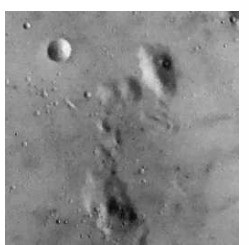

(c)

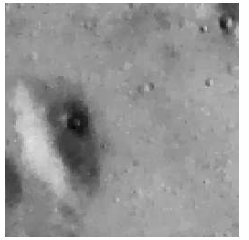

(g)

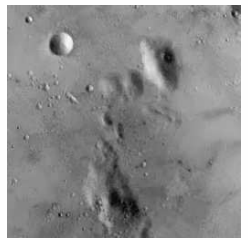

(k)

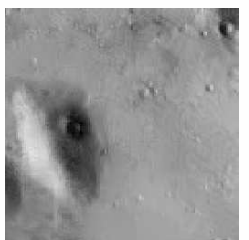

(o)

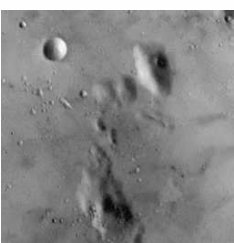

(d)

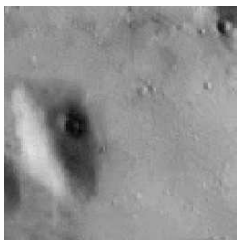

(h)

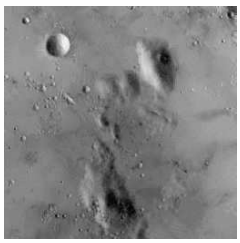

(1)

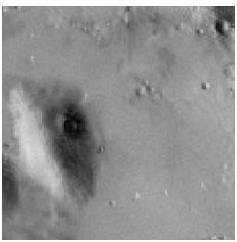

(p)

Fig. 2 Restored Moon images from $0.15 N$ measurements with 0.2 Gaussian noise. (a/e) true image/Zoom (b/f) tvqc-newton/Zoom, (c/g) NESTA/Zoom, (d/h) NLR-CS-baseline/Zoom, (i/m) NLR-CS/Zoom, (j/n) generalized HDTV/Zoom, (k/o) SRF-CS/Zoom, (l/p) Algorithm 3 Zoom.

inspection, we can observe that the proposed method and SRF-CS achieve less residual error than the other compared approaches.

Zoneplate \& Knee We respectively recover Zoneplate image from $M=0.29 \mathrm{~N}$ (80 radial lines) measurements and Knee image from $M=0.35 N$ (100 radial lines) measurements by pseudo-radial sampling their Fourier transform coefficients. The restored Zoneplate images with the tvqc-newton, NESTA and NLR-CS methods are of poor quality, as shown in Figure 6(c), (d), and (f). We can infer that the tvqc-newton, NESTA, and NLR-CS methods are not able to compensate for the large amount of missing information. On the other 


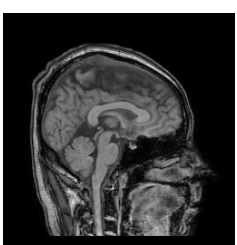

(a)

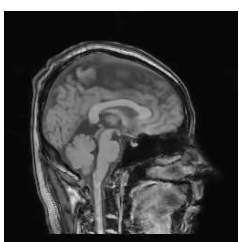

(e)

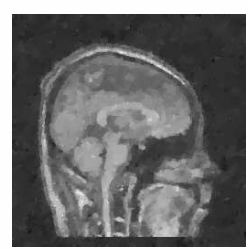

(b)

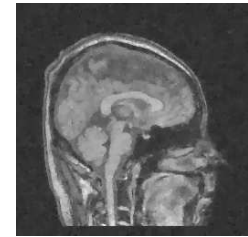

(f)

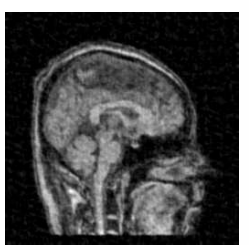

(c)

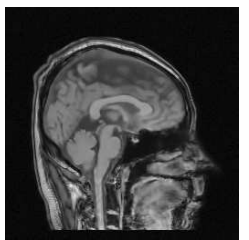

(g)

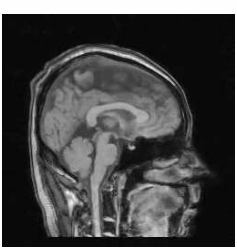

(d)

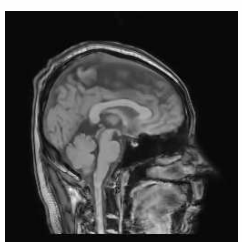

(h)

Fig. 3 Restored Head images from 0.1N measurements with 0.1 Gaussian noise. (a) true image, (b) tvqc-newton, (c) generalized HDTV, (d)NLR-CS-baseline, (e) NLR-CS, (f) NESTA, (g) SRF-CS, (h) Algorithm 3

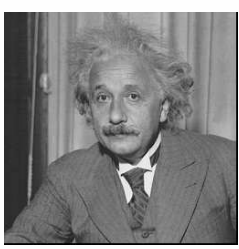

(a)

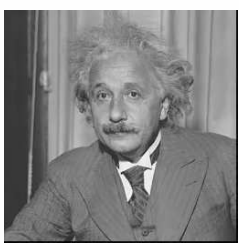

(e)

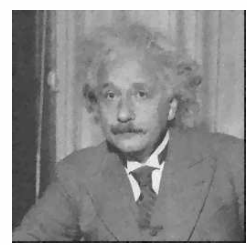

(b)

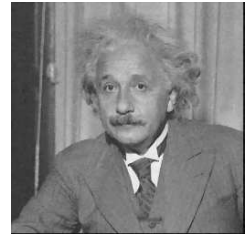

(f)

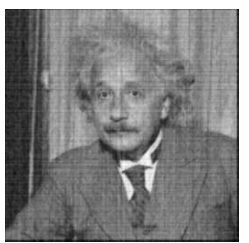

(c)

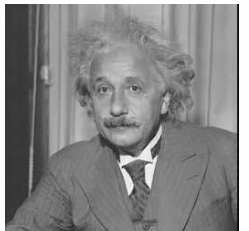

(g)

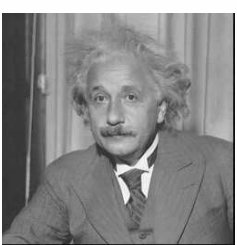

(d)

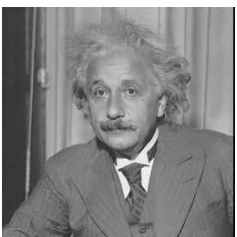

(h)

Fig. 4 Restored Einstein images from $0.2 \mathrm{~N}$ measurements with 0.3 Gaussian noise. (a) true image, (b) tvqc-newton, (c) generalized HDTV, (d)NLR-CS-baseline, (e) NLR-CS, (f) NESTA, (g) SRF-CS, (h) Algorithm 3

hand. the SRF-CS method and Algorithm 3 obtain more accurate reconstructions with respect to the other methods, as can be observed in Figure 7 For the Knee image, all the tested methods achieve similar results, as shown in Figure 8, however, as in the previous examples, the SRF-CS method and Algorithm 3 obtain the highest PSNR and SSIM values.

As above, we present the PSNR and SSIM values for all tested methods in Table 1 and Table 2. We observe that both the PSNR and SSIM values obtained with the SRF-CS method and the proposed Algorithm B are very 


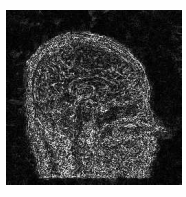

(a)

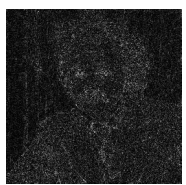

(f)

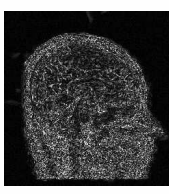

(b)

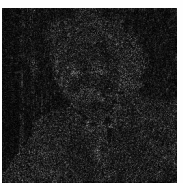

(g)

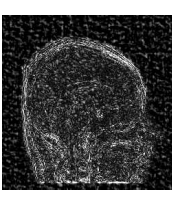

(c)

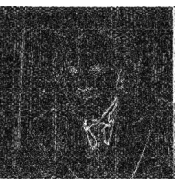

(h)

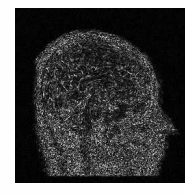

(d)

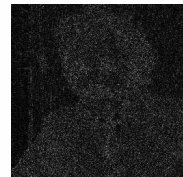

(i)

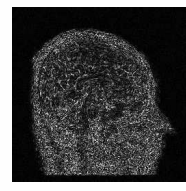

(e)

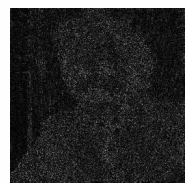

(j)

Fig. 5 Residual images: First row is related to the Head image, while the Second row is related to the Einstein image. (a,f) NLR-CS-baseline, (b,g) NLR-CS, (c,h) generalized HDTV, (d,i) SRF-CS, (e,j) Algorithm 3

\begin{tabular}{|c|c|c|c|c|c|}
\hline Image & $\begin{array}{l}\text { Number of } \\
\text { measurements }\end{array}$ & $\begin{array}{l}\text { Noise } \\
\text { level }\end{array}$ & Method & PSNR (dB) & SSIM \\
\hline Number & $\mathrm{M}=0.05 \mathrm{~N}$ & 0.15 & $\begin{array}{l}\text { tvqc-newton } \\
\text { NESTA } \\
\text { NLR-CS-baseline } \\
\text { NLR-CS } \\
\text { generalized HDTV } \\
\text { SRF-CS } \\
\text { Algorithm } 3\end{array}$ & $\begin{array}{l}10.00 \\
15.32 \\
22.08 \\
20.77 \\
17.04 \\
\mathbf{2 4 . 8 7} \\
24.61\end{array}$ & $\begin{array}{l}0.5552 \\
0.5228 \\
0.7910 \\
0.7290 \\
0.3864 \\
0.7702 \\
\mathbf{0 . 8 4 0 5} \\
\end{array}$ \\
\hline Moon & $\mathrm{M}=0.15 \mathrm{~N}$ & 0.2 & $\begin{array}{l}\text { tvqc-newton } \\
\text { NESTA } \\
\text { NLR-CS-baseline } \\
\text { NLR-CS } \\
\text { generalized HDTV } \\
\text { SRF-CS } \\
\text { Algorithm } 3 \\
\end{array}$ & \begin{tabular}{l|}
27.08 \\
30.11 \\
31.42 \\
31.21 \\
29.89 \\
$\mathbf{3 2 . 1 2}$ \\
32.02 \\
\end{tabular} & $\begin{array}{l}0.6623 \\
0.7121 \\
0.7723 \\
0.7621 \\
0.7178 \\
\mathbf{0 . 7 8 8 7} \\
0.7774 \\
\end{array}$ \\
\hline Head & $\mathrm{M}=0.1 \mathrm{~N}$ & 0.1 & $\begin{array}{l}\text { tvqc-newton } \\
\text { NESTA } \\
\text { NLR-CS-baseline } \\
\text { NLR-CS } \\
\text { generalized HDTV } \\
\text { SRF-CS } \\
\text { Algorithm } 3\end{array}$ & $\begin{array}{l}14.33 \\
18.33 \\
30.50 \\
31.93 \\
25.89 \\
\mathbf{3 2 . 4 3} \\
32.31\end{array}$ & $\begin{array}{l}0.3357 \\
0.6834 \\
0.8643 \\
0.9207 \\
0.5970 \\
\mathbf{0 . 9 5 5 8} \\
0.9493\end{array}$ \\
\hline Einstein & $\mathrm{M}=0.2 \mathrm{~N}$ & 0.3 & $\begin{array}{l}\text { tvqc-newton } \\
\text { NESTA } \\
\text { NLR-CS-baseline } \\
\text { NLR-CS } \\
\text { generalized HDTV } \\
\text { SRF-CS } \\
\text { Algorithm } 3\end{array}$ & $\begin{array}{l}20.71 \\
25.36 \\
35.70 \\
36.45 \\
27.65 \\
\mathbf{3 6 . 6 2} \\
36.60 \\
\end{array}$ & $\begin{array}{l}0.7623 \\
0.4770 \\
0.9110 \\
0.9242 \\
0.6971 \\
0.9253 \\
\mathbf{0 . 9 2 9 3}\end{array}$ \\
\hline
\end{tabular}

Table 1 The PSNR (dB) and SSIM values of the restoration results with random sampling pattern obtained by different tested approaches. 


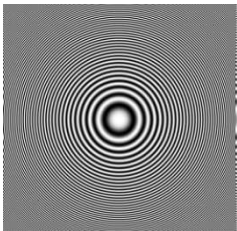

(a)

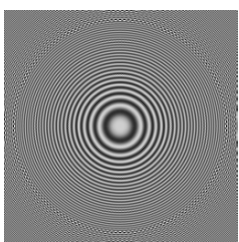

(e)

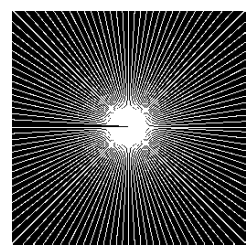

(b)

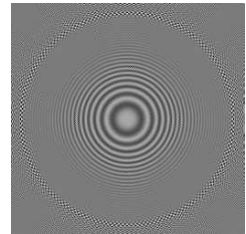

(f)

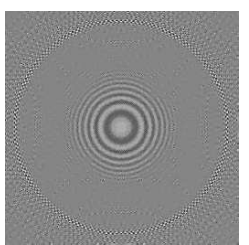

(c)

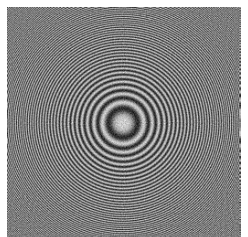

(g)

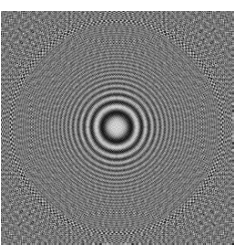

(d)

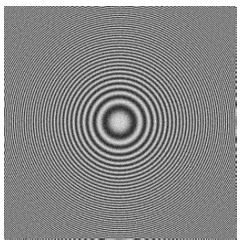

(h)

Fig. 6 Restored Zoneplate images from $0.29 \mathrm{~N}$ measurements with 0.1 Gaussian noise. (a) true image, (b) pseudo-radial sampling with 80 radial lines, (c) tvqc-newton, (d) NESTA, (e) NLR-CS-baseline, (f) NLR-CS, (g) SRF-CS, (h) Algorithm 3

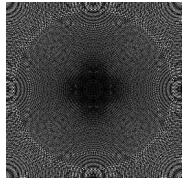

(a)

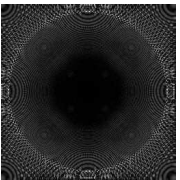

(b)

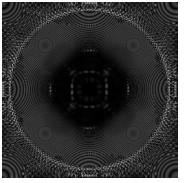

(c)

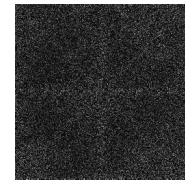

(d)

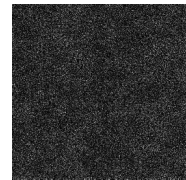

(e)

Fig. 7 Residual images of Zoneplate. (a) NESTA, (b) NLR-CS-baseline, (c) NLR-CS, (d) SRF-CS, (e) Algorithm 3

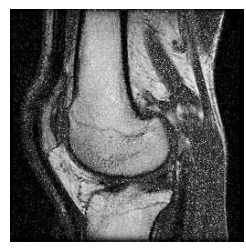

(a)

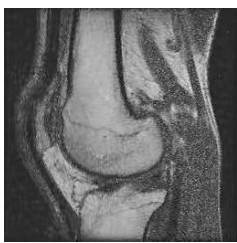

(e)

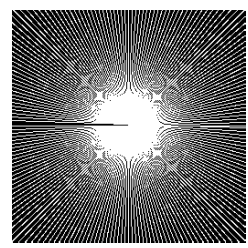

(b)

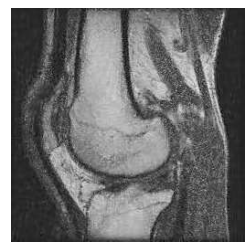

(f)

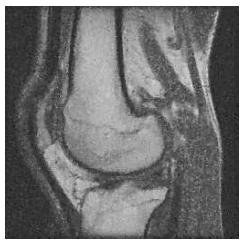

(c)

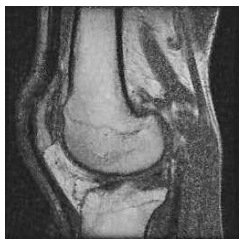

(g)

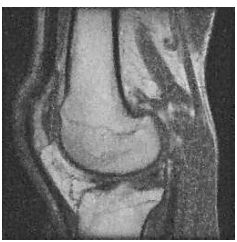

(d)

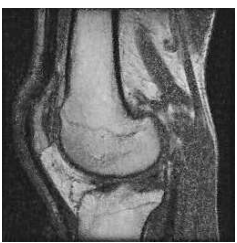

(h)

Fig. 8 Restored Knee image from $0.35 N$ measurements with 0.2 Gaussian noise. (a) true image, (b) pseudo-radial sampling with 100 radial lines, (c) tvqc-newton, (d) NESTA, (e) NLR-CS-baseline, (f) NLR-CS, (g) SRF-CS, (h) Algorithm 3 


\begin{tabular}{|c|c|c|c|c|c|}
\hline Image & $\begin{array}{l}\text { Number of } \\
\text { measurements }\end{array}$ & $\begin{array}{l}\text { Noise } \\
\text { level }\end{array}$ & Method & PSNR (dB) & $\overline{\text { SSIM }}$ \\
\hline Zoneplate & $\mathrm{M}=0.25 \mathrm{~N}$ & 0.1 & $\begin{array}{l}\text { tvqc-newton } \\
\text { NESTA } \\
\text { NLR-CS-baseline } \\
\text { NLR-CS } \\
\text { SRF-CS } \\
\text { Algorithm } 3\end{array}$ & $\begin{array}{l}9.03 \\
10.52 \\
11.30 \\
10.23 \\
\mathbf{2 4 . 5 5} \\
24.52\end{array}$ & $\begin{array}{l}0.2608 \\
0.4504 \\
0.5589 \\
0.4524 \\
\mathbf{0 . 9 5 9 9} \\
0.9564\end{array}$ \\
\hline Knee & $\mathrm{M}=0.35 \mathrm{~N}$ & 0.2 & $\begin{array}{l}\text { tvqc-newton } \\
\text { NESTA } \\
\text { NLR-CS-baseline } \\
\text { NLR-CS } \\
\text { SRF-CS } \\
\text { Algorithm } 3\end{array}$ & $\begin{array}{l}23.62 \\
23.64 \\
23.81 \\
23.88 \\
\mathbf{2 4 . 2 6} \\
24.24\end{array}$ & $\begin{array}{l}0.6511 \\
0.6513 \\
0.6607 \\
0.6659 \\
\mathbf{0 . 6 8 5 5} \\
0.6854\end{array}$ \\
\hline
\end{tabular}

Table 2 The PSNR (dB) and SSIM values of the restoration results with pseudo-radial sampling pattern obtained by different tested methods.

similar. we can then confirm that the introduction of the new terms in the model does not deteriorate the quality of the obtained reconstructions, while it allows us to provide a theoretical framework of the model and a proof of convergence of Algorithm 3 ,

\section{Conclusions}

In this paper, we have proposed a non-convex functional for compressive sensing image recovery which is able to measure the self-similarity of an image. To this aim, following [19, we have translated the self-similarity of an image into a low-rank property of a properly constructed matrix. However, since rank minimization is a NP hard problem, a non-convex smoothed function has been used as a surrogate for the rank. In [19] it has been shown that this surrogate function is a better approximation of the rank than the nuclear norm and the logdet function. On the other hand, the SRF-CS method proposal in [19] aims at minimizing a non-convex functional, which is not supported by any theoretical analysis, the existence of minimizers of this functional like the convergence of the algorithm itself have never been proved. Therefore, we have modified the model in [19] providing a theoretical analysis of the proposed functional: we have shown the existence of global minimizers, their stability, and we have proven that the minimization of this functional naturally induces a regularization method (if the involved parameters are chosen accordingly to the noise level). We have then developed an iterative algorithm for the computation of a stationary point of this non-convex functional and we have proven its convergence. Numerical experiments have shown that the proposed method achieves very similar results to the SRF-CS method obtaining sometimes a higher SSIM. Thanks to the theoretical analysis the robustness of our algorithm is guaranteed also for other examples while the same does not hold with the SRF-CS method. 
Future research should include the development of strategies for the estimation of the parameters involved, using for instance criteria like the discrepancy principle and the L-curve and a theoretical study of the exact reconstruction properties of the proposed algorithm.

\section{Acknowledgment}

The authors would like to thank the editor and the anonymous referees for their insightful comments that greatly improved the readability and the general quality of this paper.

\section{References}

1. Adcock, B., Hansen, A.C.: Generalized sampling and infinite-dimensional compressed sensing. Foundations of Computational Mathematics 16(5), 1263-1323 (2016)

2. Adcock, B., Hansen, A.C., Poon, C., Roman, B.: Breaking the coherence barrier: A new theory for compressed sensing. In: Forum of Mathematics, Sigma, vol. 5. Cambridge University Press (2017)

3. Beck, A., Teboulle, M.: A fast iterative shrinkage-thresholding algorithm for linear inverse problems. SIAM Journal on Imaging Sciences 2(1), 183-202 (2009)

4. Becker, S., Bobin, J., Candes, E.J.: Nesta: A fast and accurate first-order method for sparse recovery. SIAM Journal on Imaging Sciences 4(1), 1-39 (2011)

5. Boyd, S., Parikh, N., Chu, E., Peleato, B., Eckstein, J.: Distributed optimization and statistical learning via the alternating direction method of multipliers. Found. Trends Mach. Learn. 3(1), 1-122 (2011). DOI 10.1561/2200000016. URL http://dx.doi.org/10.1561/2200000016

6. Cai, J., Candès, E.J., Shen, Z.: A singular value thresholding algorithm for matrix completion. SIAM Journal on Optimization 20(4), 1956-1982 (2010)

7. Cai, J.F., Osher, S., Shen, Z.: Linearized bregman iterations for frame-based image deblurring. SIAM Journal on Imaging Sciences 2(1), 226-252 (2009)

8. Cai, J.F., Osher, S., Shen, Z.: Split bregman methods and frame based image restoration. Multiscale modeling \& simulation 8(2), 337-369 (2009)

9. Candes, E., Romberg, J.: $\ell_{1}$-Magic: Recovery of sparse signals via convex programming. URL https://statweb.stanford.edu/ candes/l1magic/

10. Candes, E.J., Plan, Y.: A probabilistic and ripless theory of compressed sensing. IEEE transactions on information theory 57(11), 7235-7254 (2011)

11. Candès, E.J., Romberg, J., Tao, T.: Robust uncertainty principles: Exact signal reconstruction from highly incomplete frequency information. IEEE Transactions on Information Theory 52(2), 489-509 (2006)

12. Candès, E.J., Romberg, J., Tao, T.: Robust uncertainty principles: Exact signal reconstruction from highly incomplete frequency information. IEEE Transactions on information theory 52(2), 489-509 (2006)

13. Chartrand, R.: Exact reconstruction of sparse signals via nonconvex minimization. IEEE Signal Processing Letters 14(10), 707-710 (2007)

14. Dabov, K., Foi, A., Katkovnik, V., Egiazarian, K.: Image denoising by sparse 3-D transform-domain collaborative filtering. IEEE Transactions on Image Processing 16(8), 2080-2095 (2007)

15. Dai, Y.H., Yuan, Y.: A nonlinear conjugate gradient method with a strong global convergence property. SIAM Journal on optimization 10(1), 177-182 (1999)

16. Dong, W., Shi, G., Li, X., Ma, Y., Huang, F.: Compressive sensing via nonlocal low-rank regularization. IEEE Transactions on Image Processing 23(8), 3618-3632 (2014)

17. Donoho, D.L.: Compressed sensing. IEEE Transactions on Information Theory 52(4), 1289-1306 (2006) 
18. Engl, H.W., Hanke, M., Neubauer, A.: Regularization of inverse problems, vol. 375. Springer Science \& Business Media (1996)

19. Fan, Y.R., Huang, T.Z., Liu, J., Zhao, X.L.: Compressive sensing via nonlocal smoothed rank function. PloS one 11(9), e0162,041 (2016)

20. Gehm, M., John, R., Brady, D., Willett, R., Schulz, T.: Single-shot compressive spectral imaging with a dual-disperser architecture. Optics Express 15(21), 14,013-14,027 (2007)

21. Grippof, L., Sciandrone, M.: Globally convergent block-coordinate techniques for unconstrained optimization. Optimization methods and software 10(4), 587-637 (1999)

22. Gu, S., Zhang, L., Zuo, W., Feng, X.: Weighted nuclear norm minimization with application to image denoising. In: Proceedings of the IEEE Conference on Computer Vision and Pattern Recognition, pp. 2862-2869 (2014)

23. He, B., Tao, M., Yuan, X.: Alternating direction method with gaussian back substitution for separable convex programming. SIAM Journal on Optimization 22(2), 313-340 (2012)

24. Hu, Y., Ongie, G., Ramani, S., Jacob, M.: Generalized higher degree total variation (HDTV) regularization. IEEE Transactions on Image Processing 23(6), 2423-2435 (2014)

25. Huang, G., Lanza, A., Morigi, S., Reichel, L., Sgallari, F.: Majorization-minimization generalized krylov subspace methods for $\ell_{p}-\ell_{q}$ optimization applied to image restoration. BIT Numerical Mathematics pp. 1-28 (2017)

26. Lustig, M., Donoho, D., Pauly, J.M.: Sparse MRI: The application of compressed sensing for rapid MR imaging. Magnetic Resonance in Medicine 58(6), 1182-1195 (2007)

27. Lustig, M., Donoho, D.L., Santos, J.M., Pauly, J.M.: Compressed sensing MRI. IEEE Signal Processing Magazine 25(2), 72-82 (2008)

28. Ma, T.H., Huang, T.Z., Zhao, X.L.: Group-based image decomposition using 3-D cartoon and texture priors. Information Sciences 328, 510-527 (2016)

29. Mairal, J., Bach, F., Ponce, J., Sapiro, G., Zisserman, A.: Non-local sparse models for image restoration. In: 2009 IEEE 12th International Conference on Computer Vision, pp. 2272-2279 (2009). DOI 10.1109/ICCV.2009.5459452

30. Sarvotham, S., Baron, D., Wakin, M., Duarte, M.F., Baraniuk, R.G.: Distributed compressed sensing of jointly sparse signals. In: Asilomar conference on signals, systems, and computers, pp. 1537-1541 (2005)

31. Takhar, D., Laska, J.N., Wakin, M.B., Duarte, M.F., Baron, D., Sarvotham, S., Kelly, K.F., Baraniuk, R.G.: A new compressive imaging camera architecture using opticaldomain compression. In: Electronic Imaging 2006, pp. 606,509-606,509. International Society for Optics and Photonics (2006)

32. Wang, Z., Bovik, A.C., Sheikh, H.R., Simoncelli, E.P.: Image quality assessment: from error visibility to structural similarity. IEEE Transactions on Image Processing 13(4), 600-612 (2004)

33. Zhang, X., Burger, M., Bresson, X., Osher, S.: Bregmanized nonlocal regularization for deconvolution and sparse reconstruction. SIAM Journal on Imaging Sciences 3(3), $253-276(2010)$ 